\title{
Malaria Vulnerability Map Mobile System Development Using GIS-Based Decision-Making Technique
}

\author{
Jung-Yoon Kim $\mathbb{D}^{1},{ }^{1}$ Sung-Jong Eun, ${ }^{2}$ and Dong Kyun Park ${ }^{3}{ }^{3}$ \\ ${ }^{1}$ Graduate School of Game, Gachon University, 1342 Seongnam Daero, Sujeong-Gu, Seongnam-Si, \\ Gyeonggi-Do 461-701, Republic of Korea \\ ${ }^{2}$ Health IT Research Center, Gil Medical Center, Gachon University College of Medicine, Incheon, Republic of Korea \\ ${ }^{3}$ Department of Gastrointestinal Medicine, Gil Medical Center, Gachon University College of Medicine, Incheon, \\ Republic of Korea
}

Correspondence should be addressed to Dong Kyun Park; pdk66@gilhospital.com

Received 18 June 2018; Accepted 2 August 2018; Published 18 September 2018

Academic Editor: Jaegeol Yim

Copyright (c) 2018 Jung-Yoon Kim et al. This is an open access article distributed under the Creative Commons Attribution License, which permits unrestricted use, distribution, and reproduction in any medium, provided the original work is properly cited.

\begin{abstract}
This paper aimed at improving the lack of GIS information use and compatibility of multiplatform which represented limits that existing malaria risk analysis tools had. For this, the author developed mobile web-based malaria vulnerability map system using GIS information. This system consists of system database construction, malaria risk calculation function, visual expression function, and website and mobile application. This system was developed based on Incheon region only. Database includes information on air temperature and amount of precipitation as well. With regard to malaria risk calculation, guideline provided by Korea Centers for Disease Control and Prevention was followed first and then decision-making technique was used. Calculating criteria value for risk index made it possible to estimate more precise risk. With regard to visual expression function, database constructed earlier and risk information were linked to print out graphic map and graphs so that more intuitive and visible expression can be provided based on animation technique. This system allows a user to check information in real time and can be used anywhere anyplace. Mobile push function is to enhance user's convenience. Such web map is useful to general users as well as experts.
\end{abstract}

\section{Introduction}

Malaria is infectious disease which is mediated and disseminated by Anopheles sinensis. Malaria is caused by Plasmodium (Plasmodium genus) which penetrates in red blood cell. Malaria shows symptom of fever, chill, hepatomegaly, splenomegaly, and so on. Malaria is reported to have occurred in one hundred four countries in the world. It is said that over one billion people around the world are exposed to a danger of malaria and over three hundred million people are infected with malaria and over one million people die of malaria on a yearly basis [1]. Plasmodium is Plasmodium falciparum, $P$. vivax, $P$. malariae, $P$. ovale, and $P$. knowlesi. It is said that malaria occurs with mosquito as mediator. It is reported that in South Korea, mainly vivax malaria caused by $P$. vivax which is mediated by Anopheles genus mosquito has occurred. As from 1979, South Korea was declared as malaria-free area. However, in 1993, vivax malaria occurred in soldiers on the front line who served in the army near North Korea. It is reported that from 1993 to 2013, 500-4,000 malaria cases occurred centering on DMZ (demilitarized zone) annually. Vivax malaria which reoccurred in 1993 has appeared for about twenty years in northern part of Gyeonggi Province and varied in frequency of occurrence. Until now, vivax malaria occurred with three times of peaks (1998 2000, 2007, and 2010, respectively). It is reported that malaria cases have continued to occur until 2017 [1-3]. Figure 1 shows occurrence of malaria in South Korea by years.

Malaria is infectious disease that needs continuous control. Scholars have conducted studies on the cause of malaria and incidence rate by correlation considering meteorological elements and community characters [4-6]. In addition, they 


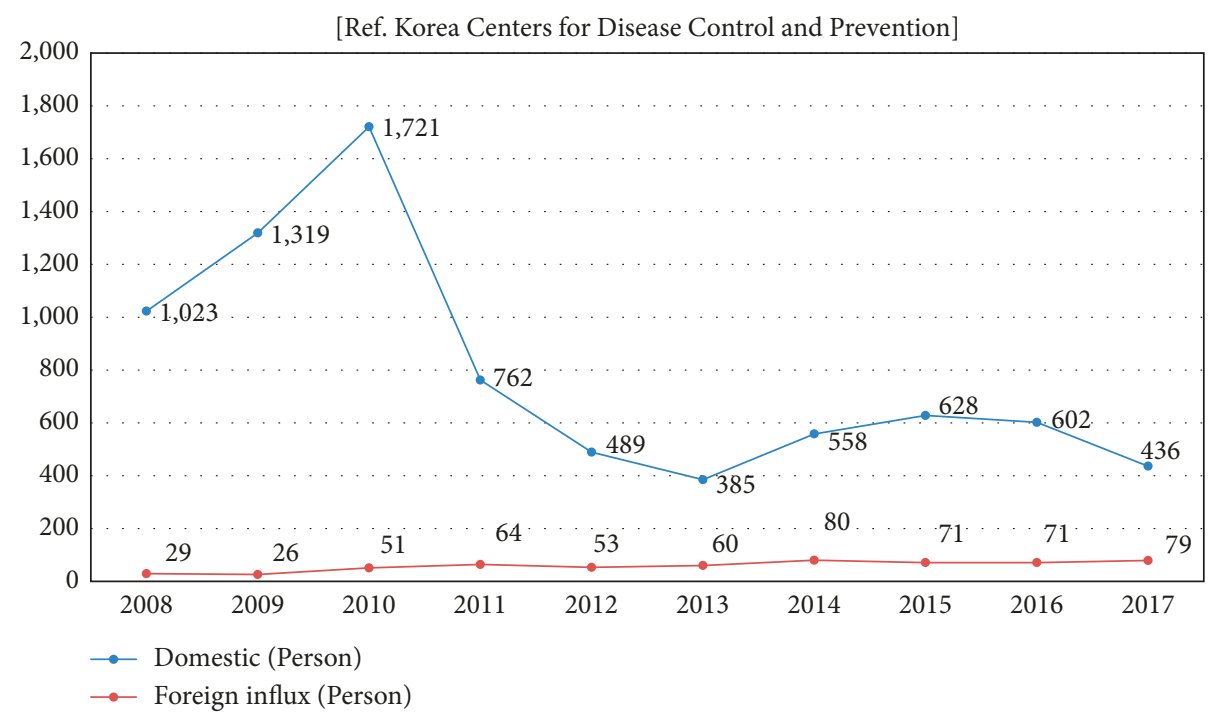

Figure 1: Annual malaria infection status.

analyzed route of infection in terms of epidemiology or environmental factors to prevent occurrence of malaria.

Software tool [7] that supports analysis of malaria-related information is being developed. As quantity of data becomes increased, applying technique of processing big data to correlation analysis is being raising with care. Like this, in health science, studies have been conducted in a specialized way making good use of IT-related technologies. Database in the field of health science includes a variety of data most of which is obvious in terms of space or refers to space. Therefore, database in the field of health science has the advantage of using data in GIS. Diversity, size, and complexity of health science data need more application of GIS [8]. Field of controlling infectious disease such as malaria needs GIS-based tools for risk analysis. This is to make good use of GIS-based analysis that can analyze information in an intuitive and visible way. Such tools have been developed, but they are not widely used due to problem of compatibility of independent platform, problem of exclusive institutional policy, and limited information.

This paper built malaria vulnerability map system using GIS information-based decision-making technique in order to limit problems of abovementioned tools and compatibility of multiplatform. Malaria vulnerability map system drew wellfounded resulting values by making good use of various data on air temperature, humidity, amount of precipitation, lakes, and population with GIS information. Malaria vulnerability map system was built based on mobile web to enhance users' convenience. In terms of UX/UI, animation function helps enhance users' immersion and accessibility by providing intuitive information. This study consists of present condition of relevant tool, content of development system, conclusion, and investigation.

\section{Related Works}

Tools that analyze risk of malaria have been developed at home and abroad. In South Korea, abovementioned tools are used in some institutions for internal use only. In foreign countries, MDAST (Malaria Decision Analysis Support Tool) project is being carried out. MDAST project is developing a tool that analyzes a cause of occurrence of malaria in Kenya, Tanzania, and Uganda where malaria occurs frequently and predicts incidence rate [9].

MDAST aims to provide a platform to analyze influences in terms of health, society, and environment for institution that establishes and evaluates malaria-related policy by using various scientific methods of analyzing information in malaria. This service downloads a tool. Sets it up on local, enters relevant data, sets several factors, applies desired analysis methods, and obtains a result. Analysis methods support various statistical analysis methods. Above tools are widely used but require professional knowledge in using such as factor setting and analysis method selection and impossible to provide online-based real-time support. The major drawback of above tools is that it is difficult for general user to access.

In South Korea, above tools are used through intranet or local installation by health-care-related institutions. They are used by some institutions for their internal use. Since the early 2000, some institutions have developed analysis tools. Environmental factors such as weather and geography and weights are selected on a case-by-case basis to handle system. GIS-based analysis system predicts a risk of malaria and prints out information on a map. For above tools, setting factors of several environmental elements plays an important role. Factors are handled through user definition.

GIS-based analysis system is to cope with unexpected local occurrence of malaria and prevent malaria from spreading by detecting an area where malaria is likely to occur as soon as possible. GIS-based analysis system which is used in South Korea varies in tools among institution, which makes performance deviation for functions supported great. It is difficult to define tools which are used because they are used for internal use.

GIS-based analysis system which is used in South Korea is used by some institutions for their internal use and is based on local. There are few systems which show information on 
environmental elements relating to malaria on the website. This study developed map system which delivers GIS-based information on risk of malaria on mobile website in consideration of the foregoing. System was built in Incheon region. DB was built by receiving data from institutions such as Korea Meteorological Administration and K-Weather. This study built web-based malaria vulnerability map system so that general users can be provided with information on malaria risk by region in real time.

\section{Developing Malaria Vulnerability Map Mobile Using GIS-Based Decision- Making Technique}

The purpose of this paper was to develop a map that can analyze factors which cause malaria by using mobile webbased GIS (Geographic Information System). Decision tree analysis method was applied to model areas where malaria is more likely to occur. GIS and decision-making system to develop Korean-type malaria vulnerability map were finally built.

A map of this system was made based on overlay operation method that can provide new information which is not identified on respective map by combining several maps. Population, humidity, temperature, amount of precipitation, and lakes were considered for decision-making technique. Weight was calculated by using AHP decision-making method. Final malaria vulnerability map was made by using PROMETHEE technique.

Map system was implemented based on mobile web for compatibility with mobile platform. For web-based visual output, frame animation-based reading of areas where malaria appeared, reading of summarized information on areas where malaria appeared, reading of detailed information on areas where malaria appeared, and display of risk according to areas where malaria appeared were expressed visually.

3.1. Details of System Development. System functions consist of system database, malaria risk calculation, visual expression, and website and mobile application. Detail core functions are decision-making technique-based modelling part for malaria vulnerability calculation and mobile web map construction part for visual expression and program extension. The system configuration is shown in Figure 2.

3.1.1. Building Up System Database. Web-based system and MySQL with good compatibility and proven stability were used as database system to organize database.

Database information specific to Incheon region consists of data from 2011 to 2017. Area code was extracted based on longitude and latitude to provide information specific to Incheon so that it can provide detailed information according to region.

Information consisting of DB includes population, humidity, air temperature, amount of precipitation, and lake in Incheon. Information on lakes was used in determining vulnerability because whether there is mosquito can be a factor on which high weight for malaria risk can be placed. With regard to information on air temperature, relative humidity, and amount of precipitation, data provided by Korea Meteorological Administration and K-Weather were used. Other information was provided by Incheon Institute of Health Environment. For relevant DB, schema was defined in one table without dividing it into several tables according to characteristics of data to cut down on expense caused by reference between tables and extract database information rapidly. Table 1 shows the structure of the system DB, which shows the structure of information on Anopheles sinensis, region code, sex, temperature, relative humidity, and precipitation.

For system database organized, unlike existing analysis tools where information on air temperature or amount of precipitation was not provided, various environmental factors were considered.

\subsubsection{Developing a Model for Calculating Malaria Risk}

(1) Calculating optimum weight using AHP (analytic hierarchy process) technique. Malaria risk is calculated based on various standards. For risk modelling work, various weights are considered. In this paper, population, humidity, air temperature, amount of precipitation, and lake were considered. Weight for each criterion was reflected based on experts' opinion as importance of standards differs in creating malarial risk. For this, AHP (analytic hierarchy process) was used. AHP [10-12] is a calculation model which was devised based on the fact that brain uses stepwise or hierarchical analysis process. AHP reaches a final decision making by dividing whole process of decision making into multiphases and analyzing it on step-by-step basis. The author of this paper carried out AHP based on flowchart as shown in Figure 3.

Order of standards which were considered based on Figure 3 was developed as shown in Figure 4. In phase 2 of order of rank, comparison of standards was made based on judgment of experts in malaria [13]. Table 2 shows comparison of pairs. Lastly, weight for standards was calculated by using eigenvector and eigenvalue in each comparison matrix.

Final weight criteria estimated through AHP decisionmaking technique were calculated based on temperature of 0.51 , humidity of 0.34 , population of 0.1 , and lake of 0.15 .

(2) Calculating decision-making matrix using PROMETHEE technique. Methods that select the best alternative by objectively measuring preference for alternatives considered under each criterion have been studied a lot. Typical techniques are ELECTRE and PROMETHEE. ELECTRE I, II, III, and IV technique proposed by B. Roy can solve difficult problems well and is the most reliable method, but the calculation process is very complex and requires decision maker to decide lots of parameters. On the other hand, PROMETHEE (Preference Ranking Organization Method Enrichment Evaluations) is very simple and easy for decision maker to understand and 


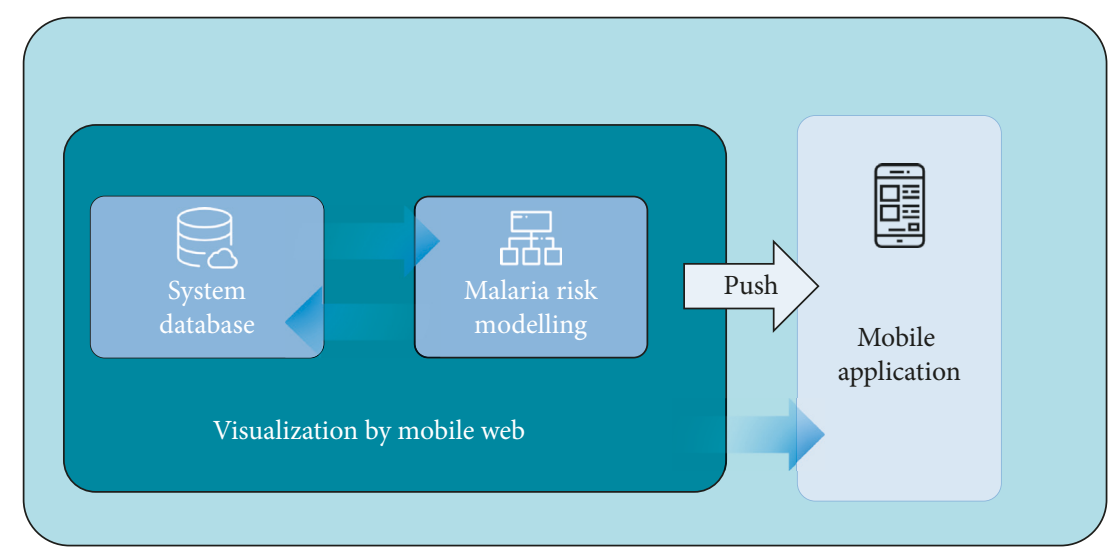

FIgURE 2: Conceptual diagram of malaria vulnerability map mobile system.

TABLE 1: External schema.

\begin{tabular}{lcc}
\hline Data & Unit & Type \\
\hline Anopheles sinensis & Count & Integer \\
Region code & - & Integer \\
Male & 1,000 persons & Integer \\
Female & 1,000 persons & Integer \\
Temperature & ${ }^{\circ} \mathrm{C}$ & Float \\
Relative humidity & $\%$ & Float \\
Amount of precipitation & $\mathrm{mm}$ & Float \\
\hline
\end{tabular}

preference intensity for alternatives is expressed with easy concept and requires decision maker to decide up to two parameters, which makes it preferred (Brans, 1985).

PROMETHEE proposed by Brans and Vincke [14] defines preference functions according to each evaluation criterion as Type I (Usual Criterion), Type II (Quasicriterion), Type III (Criterion with Linear Preference), Type IV (Level Criterion), Type V (Criterion with Linear Preference and Indifference Area), and Type VI (Gaussian Criterion) [15-19]. PROMETHEE evaluates preference relationships among alternatives by using preference index $\pi(a, b)$.

$$
\pi(a, b)=\frac{1}{k} \sum_{h=1}^{k} p_{h}(a, b),
$$

All evaluation criteria must be defined as one of the abovementioned six preference functions. $p_{h}(a, b)$ in Equation (1) represents preference function value for evaluation criteria $h$. Decision maker should decide a shape of preference function according to evaluation criteria and assign threshold according to preference functions.

The author of this paper implemented PROMETHEE for each level five times. In each repetition, $n x k$ decision-making matrix was created. $n$ is the number of points (alternatives) and $k$ is the number of criteria. Decision-making matrixnumber represents criteria for alternatives (regions). Table 3 represents decision-making matrix drawn when first time was repeated.

Decision matrix elements to be used in decisionmaking technique are decided based on the foregoing. Applicable components are decision-making matrix. Incheon region was divided into 28 areas. Matrix values to

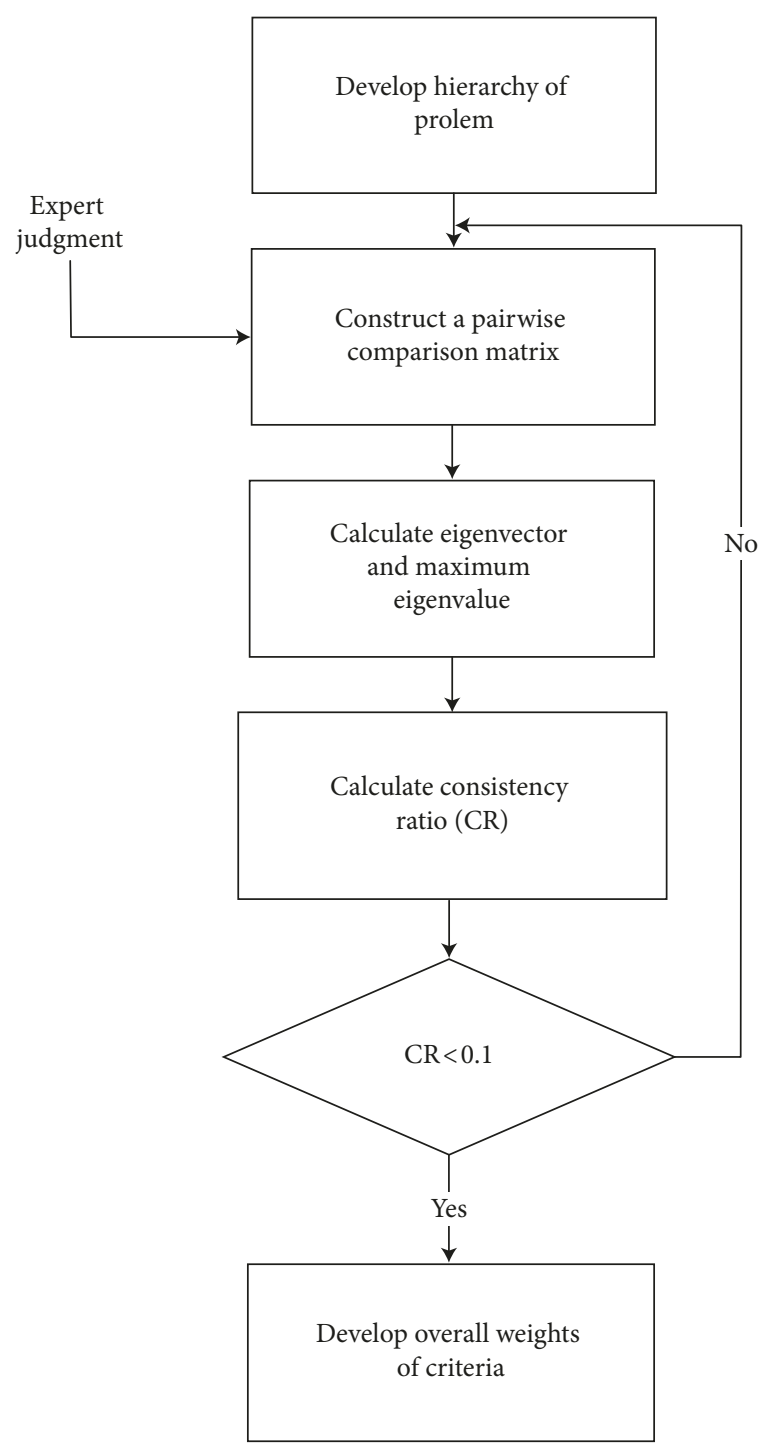

Figure 3: AHP for calculating criteria weight.

decide population, humidity, temperature, and lake were as shown above. Table 4 represents final result obtained from PROMETHEE. Five different rankings for 28 areas were obtained by implementing PROMETHEE five times. 


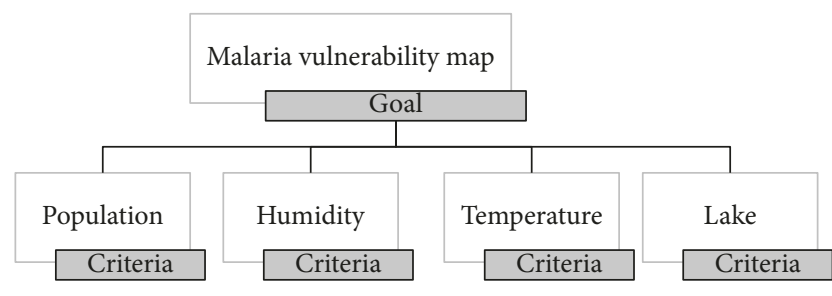

FIgURE 4: Structure of order of rank in criteria.

TABLE 2: External schema.

\begin{tabular}{lcccc}
\hline Criteria & Air temperature & Humidity & Lake & Population \\
\hline Air temperature & 1 & 3 & 3 & 4 \\
Humidity & $1 / 3$ & 1 & 2 & 2 \\
Lake & $1 / 3$ & $1 / 2$ & 1 & 2 \\
Population & $1 / 4$ & $1 / 2$ & $1 / 2$ & 1 \\
\hline
\end{tabular}

TABle 3: Decision-making matrix.

\begin{tabular}{lcccc}
\hline Zones & Population & Humidity & Temperature & Lake \\
\hline 1 & 4.085648 & 5.867935 & 1 & 4.064596 \\
2 & 6.652511 & 6 & 1.685264 & 8.64964 \\
3 & 6.11661 & 7.43814 & 8.964341 & 5 \\
4 & 4.140974 & 7.444163 & 7.649631 & 7.165594 \\
5 & 4 & 2.487076 & 1 & 0 \\
6 & 3 & 5 & 1 & 0 \\
7 & 3 & 5 & 1 & 0 \\
8 & 3.452714 & 3.34022 & 1 & 0 \\
9 & 5.655222 & 6 & 40951.15 & 6.865466 \\
10 & 8.097396 & 6 & 97781.63 & 4.169856 \\
11 & 5.438997 & 6.478203 & 1.986046 & 4.359985 \\
12 & 7.964342 & 6.408581 & 7.865646 & 3.598465 \\
13 & 7.019162 & 7.085386 & 6.131337 & 4.006419 \\
14 & 5.354643 & 8.078567 & 8.643161 & 7.645985 \\
15 & 8.989335 & 6 & 3.641969 & 4.036452 \\
16 & 1.969482 & 9 & 4.945094 & 7.264995 \\
17 & 4.945331 & 7.766397 & 5.619694 & 9.165985 \\
18 & 7.842272 & 6.142719 & 6.940964 & 4.358643 \\
19 & 3.928667 & 2.519822 & 1 & 0 \\
20 & 4.123696 & 4.496581 & 1.690846 & 2.365646 \\
21 & 4 & 3.717338 & 1 & 0 \\
22 & 4 & 2.113713 & 1 & 0 \\
23 & 3 & 5.402396 & 1 & 0 \\
24 & 3 & 5 & 1 & 0 \\
25 & 4 & 3.196849 & 1 & 0 \\
26 & 3.219948 & 3.921217 & 1 & 0 \\
27 & 3 & 4.640017 & 1 & 0 \\
28 & 3.361399 & 3.569278 & 1 & 0 \\
\hline & & & &
\end{tabular}

\subsubsection{Creating Malaria Vulnerability Map}

(1) Raster overlay operation. Overlay operation is applied to most GIS programs. Overlay operation provides new information by combining several maps. In overlay operation, new special elements are formed based on several maps. Overlay operation is handled based on raster and vector maps. Raster data structure is suitable for such operation because all maps used for analysis have the same georeference. They have the
TABLE 4: PROMETHEE index.

\begin{tabular}{lc}
\hline Zones & Population \\
\hline 1 & 14 \\
2 & 10 \\
3 & 2 \\
4 & 8 \\
5 & 21 \\
6 & 0 \\
7 & 0 \\
8 & 25 \\
9 & 11 \\
10 & 6 \\
11 & 12 \\
12 & 5 \\
13 & 4 \\
14 & 1 \\
15 & 9 \\
16 & 13 \\
17 & 3 \\
18 & 7 \\
19 & 23 \\
20 & 15 \\
22 & 17 \\
23 & 26 \\
24 & 16 \\
25 & 18 \\
26 & 19 \\
27 & 22 \\
28 & 20 \\
\hline
\end{tabular}

same number of pixels and consist of row and column and have the same pixel size and coordinate. Accordingly, when several maps are combined, a program examines each pixel and the same figures can be checked from different maps. In raster overlay, cell numbers are combined in a specific way and figures obtained are assigned to corresponding cells in output layer. Raster overlay is applied to explicit or ordinal number data. Number or characters are stored in each raster cell. Figures in each cell correspond to items of raster variables. In Figure 5, Layer A represents solid data and Layer B shows an example of raster overlay that records land use. Number of potential output items is number of combination that input items can have $(2 * 3=6)$.

Figure 6 shows number of population, humidity, temperature, lake, and vulnerability map result calculated by raster overlay. Population shape file based on areas of distribution of population has been converted to raster file. As all criteria maps must have common scale, figures of population raster file have been converted to figures of 0 to 10 by using reclassification analysis. Only reclassification analysis was implemented based on humidity data. Figure was obtained by reclassifying humidity raster file. Like humidity map, air temperature map was created by using reclassification analysis. This was handled by reclassifying figures of air temperature raster file. As distance from lake is greater, malaria risk decreased. A number of ring buffer analyzes were implemented to determine area around lake from diverse distances. By using this analysis, various buffers were created from distances of 100, 200,300,400, 600, 800, 1000, 


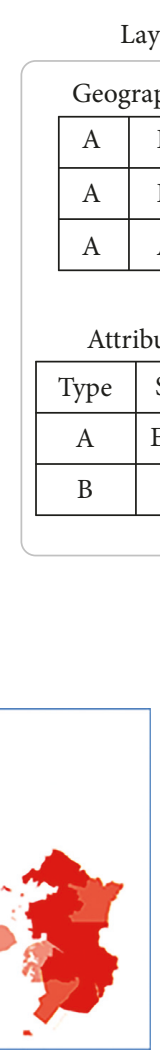

(a)

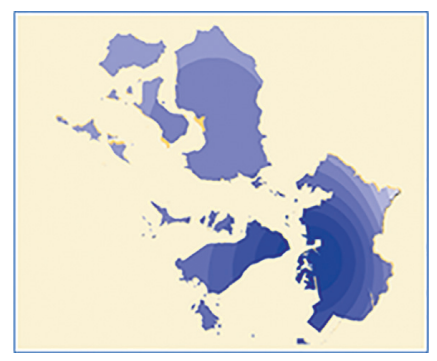

(b)

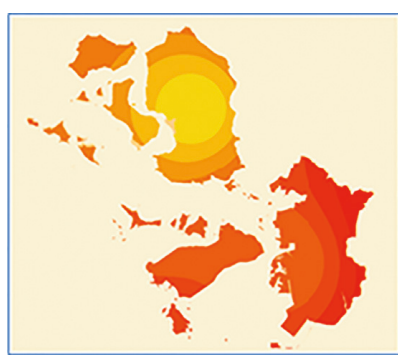

(c)

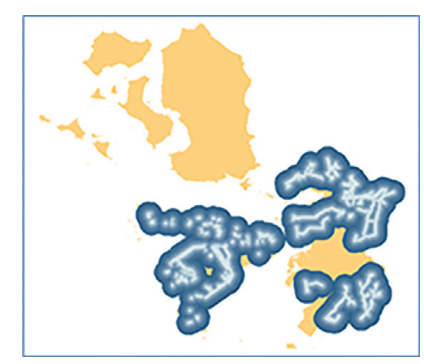

(d)

Figure 6: A result of raster overlay-based conversion and mapping. (a) Population map. (b) Humidity map. (c) Temperature map. (d) Lake map.

1500, and 2000. Buffer layers were converted to raster file based on distance area. The result that overlaps the map based on the converted raster file is shown in Figure 7.

(2) Creating malaria vulnerability map through Thiessen polygon. Incheon region was classified into 28 zones. Procedures for creating polygon were repeated five times, and 28 zones were set in Incheon Metropolitan City at random. Thiessen polygon [20] analysis was conducted by using 28 zones. Figure 7 shows Thiessen polygon created by repetition of five times.

Analysis was conducted by using mean statistics obtained by assigning mean figures in each zone to output cells. This procedure was implemented for Thiessen polygon and shows criteria map according to zones for first repetition. Accordingly, PROMETHEE was applied to criteria figures corresponding to 28 zones. Malaria risk map was created by combining risk maps obtained every time repetition was made. Repetition of applicable procedures helps in creation of more accurate malaria risk map (map closer to reality).

\subsection{Developing Mobile Web-Based Malaria Vulnerability Map Application}

3.2.1. Developing Mobile Web-Based Application. PHP is used as server development language for construction of website. Client side language was implemented as HTML, CSS3, and JavaScript (Bootstrap, jQuery). Google Maps JavaScript API v3 was used as a map. Marker was put on control area with JavaScript. When marker is selected, information contained in database is presented in graph.

Mobile application was developed as android-based hybrid web app. Push function and GPS sensor were used so that location can be tracked on background. Information on malaria rick according to periods can be provided to a user based on it. Restful API was developed through PHP so that when longitude and latitude are handed over on background, a user can check whether it entered applicable area. For push implementation, GCM (Google Cloud Message) [21, 22] service was used so that push service can be used in a stable manner irrespective of terminal's connection to network.

\subsubsection{Developing Animation-Based Visualization Technology.} Visual expression of information was implemented for intuitive and high visibility based on mobile web map. Frame animation [23], one of the animation techniques, was used for immersion. Wind direction, wind speed, spot atmospheric pressure, and sea level pressure in addition to foregoing database information were expressed in graph. Implementation functions consists of reading of areas where malaria appear, reading of summarized information on areas where malaria appear, reading of detailed 


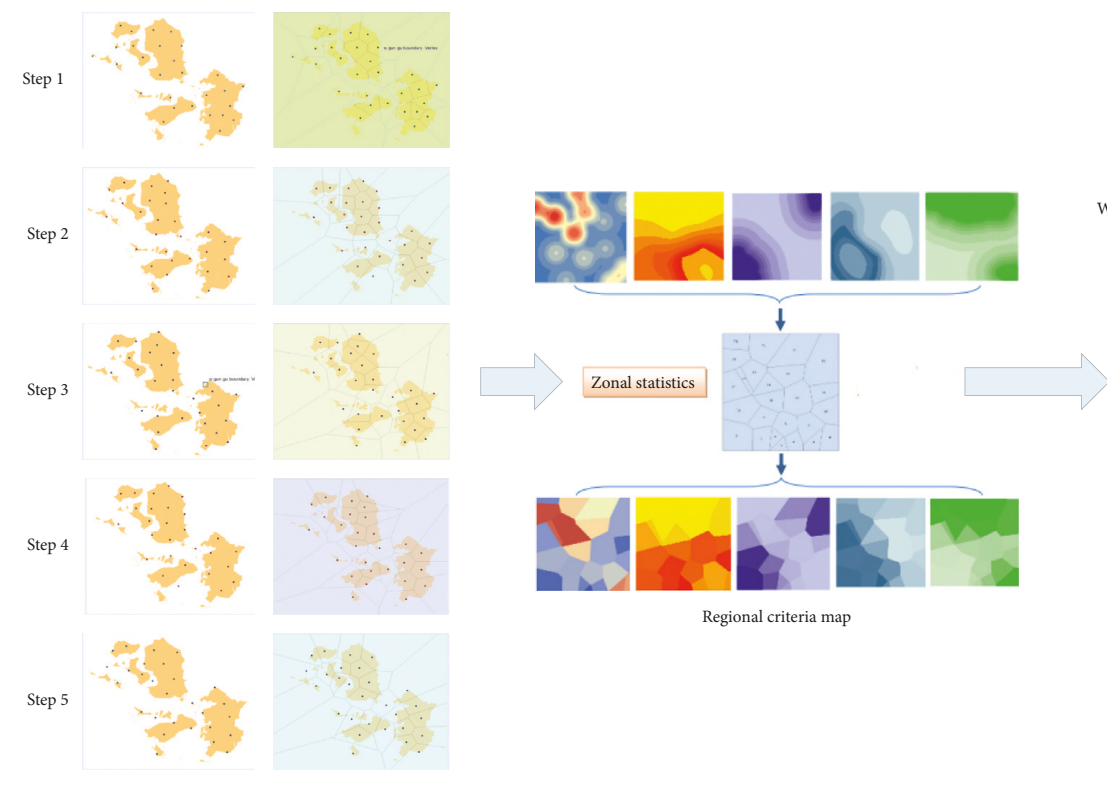

Figure 7: Raster overlay analysis.

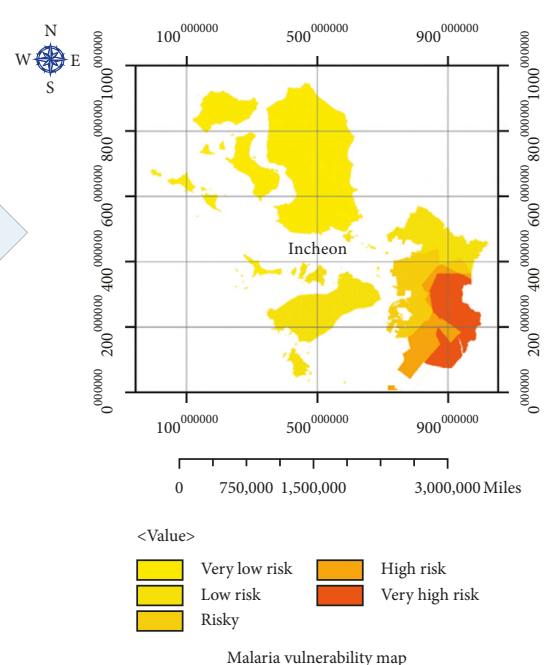

Malaria vulnerability map information on areas where malaria appear, and risk animation function for areas where malaria appear. Visual expression function was implemented by using Google Maps API and JavaScript (Bootstrap, jQuery). Frame animation technique was used for effective view of risk information. Frame animation technique can highlight specific content and provide information to a user by changing images by time and static image to dynamic image stimulating human senses which are sensitive to dynamic motions. The author of this paper attempted to convey risk and environmental information by using the abovementioned functions and make a user have easier access by supporting intuitive UI for higher level of immersion. Applicable implementations are presented in Figure 8. The DB defines the schema as shown in Table 1.

\section{Evaluation and Conclusion}

4.1. System Model Evaluation. In order to evaluate the system performance, we measured accuracy of the malaria risk model. Comparisons of accuracy criteria were compared with those of the experts' risk criteria. So we use the confusion matrix and calculate the accuracy by true positive (TP), false positive (FP), false negative (FN), and true negative (TN). Table 5 represents confusion matrix result for the evaluation of the system model.

A total of 30 test cases were used for the evaluation, which resulted in a good performance of $91.7 \%$ accuracy. Equation (2) for calculating the accuracy is processed through the following confusion matrix:

$$
\text { Accuracy }=\frac{(\mathrm{TN}+\mathrm{TP})}{(\mathrm{TN}+\mathrm{TP}+\mathrm{FN}+\mathrm{FP})} \text {. }
$$

For some inaccurate results, there is a problem in weight calculation of the AHP model to create the decision matrix.
Therefore, the issue of weight is in the order of rank in criteria. This section is classified as a special case, and more accurate weighting methods are needed. As a workaround, we expect to give more conditions to the quarter.

4.2. Conclusion. PHP is used as server development language for construction of website. Client side language was implemented as HTML, CSS3, and JavaScript (Bootstrap, jQuery). Google Maps JavaScript API v3 was used as a map.

Scholars have conducted research to develop GIS-based tools that can analyze malaria risk as continuous control of occurrence of malaria is needed. However, analysis tools at home and abroad are not widely used due to the lack of GIS information use, independent platform environment, and exclusive institutional policies.

This paper aimed at improving the lack of GIS information use and compatibility of multiplatform which represented limits that existing malaria risk analysis tools had. For this, the author developed mobile webbased malaria vulnerability map system using GIS information.

This system consists of system database construction, malaria risk calculation function, visual expression function, and website and mobile application. This system was developed based on Incheon region only. Database includes information on air temperature and amount of precipitation as well. With regard to malaria risk calculation, guideline provided by Korea Centers for Disease Control and Prevention was followed first and then decision-making technique was used. Calculating criteria value for risk index made it possible to estimate more precise risk. With regard to visual expression function, database constructed earlier and risk information were linked to print out graphic map and graphs so that more intuitive and visible expression can 


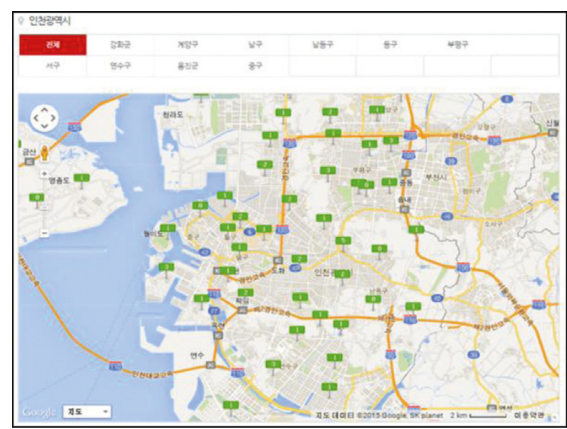

(a)

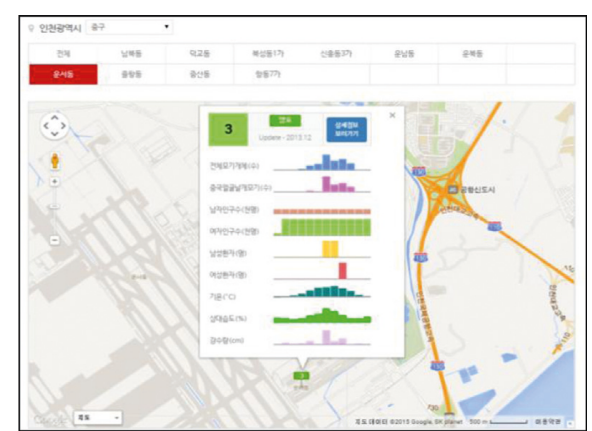

(b)

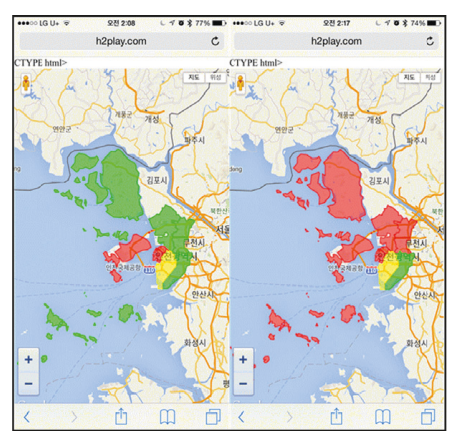

(c)

Figure 8: Final result of system visualization. (a) Marking of malaria generation region. (b) Summation information (tooltip) of malaria generation region. (c) Risk time-series animation of malaria generation region.

TABLE 5: Confusion matrix result for evaluation.

\begin{tabular}{lc}
\hline Confusion matrix & Malaria risk model \\
\hline True positive & 27 \\
False positive & 3 \\
True negative & 28 \\
False negative & 2 \\
\hline
\end{tabular}

be provided based on animation technique. This system allows a user to have easier access via website and mobile application which print out above information. This system allows a user to check information in real time and can be used anywhere anyplace. Mobile push function is to enhance user's convenience. Such web map is useful to general users as well as experts.

As an evaluation of system model, we can check the good performance of $91.7 \%$ accuracy. However, for some inaccurate results, there is a problem in weight calculation of the AHP model to create the decision matrix. This section is classified as a special case, and more accurate weighting methods are needed. To solve the issue, we expect to give more conditions to calculate the weight value.

This system is expected to be used for institutions that operate under exclusive policy and service that prints out information based on web map. This system is expected to be used as basic technology that provides visible and intuitive information in the field of infographics which offer location-based information, GIS, industries as well as health care. The author of this paper will consider environmental elements which were not used in analyzing this system and seek additional mobile functions and a way to enhance user's accessibility. And we will also consider network security technologies for securing user data [24-27].

\section{Data Availability}

Previously reported geographic information data were used to support this study and are available at Korea Meteorological Administration, K-Weather, and Incheon Metropolitan City Institute of Health and Environment. These prior studies (and datasets) are cited at relevant places within the text as references $[7,8]$.

\section{Conflicts of Interest}

The authors declare that there are no conflicts of interest regarding the publication of this paper.

\section{Acknowledgments}

This research was supported by the Bio \& Medical Technology Development Program of the National Research Foundation (NRF) funded by the Ministry of Science, ICT and Future Planning (2017M3A9E2072689).

\section{References}

[1] J. Y. Kim, "Assessments on trend of malaria prevalence in Republic of Korea," Public Health Weekly Report, vol. 7, no. 12, pp. 237-242, 2014.

[2] J.-W. Park, "Status of vivax malaria in the Republic of Korea," Journal of the Korean Medical Association, vol. 47, no. 6, pp. 521-526, 2004.

[3] Korea Center For Disease Control and Prevention, Infectious Diseases Surveillance Yearbook, Ministry of Health and Welfare, Korea Center For Disease Control and Prevention, Cheongju-si, South Korea, 2014.

[4] H. S. Shin, "Malaria prevalence rate and weather factors in Korea," Health and Social Welfare Review, vol. 31, no. 1, pp. 217-237, 2011.

[5] S. M. Chae, D. J. Kim, S. J. Yoon, and H. S. Shin, "The impact of temperature rise and regional factors on malaria risk," Health and Social Welfare Review, vol. 34, no. 1, pp. 436-455, 2014.

[6] J. Kwak, J. Lee, H. Han, and H. Kim, “A case study: malaria modeling based on climate variables in Korea," Health and Social Welfare Review, vol. 33, no. 4, pp. 547-569, 2013.

[7] Y. W. Gong, "Design and implementation of outbreak estimation system for infectious disease using GIS (for malaria)," Engineering Master thesis, Kyungwon University, Gyounggido, Republic of Korea, 2001.

[8] R. Laurini, Information Systems for Urban Planning: A Hypermedia Cooperative Approach, CRC Press, Boca Raton, FL, USA, 2014.

[9] http://sites.duke.edu/mdast/. 
[10] T. L. Saaty, "Analytic hierarchy process," in Encyclopedia of Operations Research and Management Science, pp. 52-64, Springer, Boston, MA, USA, 2013.

[11] O. S. Vaidya and S. Kumar, "Analytic hierarchy process: an overview of applications," European Journal of operational research, vol. 169, no. 1, pp. 1-29, 2006.

[12] S. H. Hashemi, A. Karimi, and M. Tavana, "An integrated green supplier selection approach with analytic network process and improved Grey relational analysis," International Journal of Production Economics, vol. 159, pp. 178-191, 2015.

[13] Korea Center for Disease Control and Prevention, Malaria Administrative Guideline 2013, Korea Center for Disease Control and Prevention, Cheongju-si, South Korea, 2013.

[14] J. P. Brans and and Ph. Vincke, "Note-A Preference Ranking Organisation Method: (The PROMETHEE Method for Multiple Criteria Decision-Making)," Management science, vol. 31, no. 6, pp. 647-656, 1985.

[15] J. Rezaei, "Best-worst multi-criteria decision-making method," Omega, vol. 53, pp. 49-57, 2015.

[16] J.-J. Wang and D.-L. Yang, "Using a hybrid multi-criteria decision aid method for information systems outsourcing," Computers \& Operations Research, vol. 34, no. 12, pp. 36913700, 2007.

[17] C. Kahraman, S. C. Onar, and B. Oztaysi, "Fuzzy multicriteria decision-making: a literature review," International Journal of Computational Intelligence Systems, vol. 8, no. 4, pp. 637-666, 2015.

[18] M. Dağdeviren, "Decision making in equipment selection: an integrated approach with AHP and PROMETHEE," Journal of Intelligent Manufacturing, vol. 19, no. 4, pp. 397-406, 2008.

[19] A. Mardani, A. Jusoh, K. M. Nor, Z. Khalifah, N. Zakwan, and A. Valipour, "Multiple criteria decision-making techniques and their applications: a review of the literature from 2000 to 2014," Economic Research-Ekonomska Istraživanja, vol. 28, no. 1, pp. 516-571, 2015.

[20] P. L. Ibisch, M. T. Hoffmann, S. Kreft et al., "A global map of roadless areas and their conservation status," Science, vol. 354, no. 6318, pp. 1423-1427, 2016.

[21] Y. S. Yilmaz, B. I. Aydin, and M. Demirbas, "Google cloud messaging (GCM): an evaluation," in Proceedings of Global Communications Conference (GLOBECOM), pp. 2807-2812, Austin, TX, USA, December 2014.

[22] N. Saravanan, A. Mahendiran, N. V. Subramanian, and N. Sairam, "An implementation of RSA algorithm in Google cloud using cloud SQL," Research Journal of Applied Sciences, Engineering and Technology, vol. 4, no. 19, pp. 3574-3579, 2012.

[23] M. Achibet, G. Casiez, A. Lécuyer, and M. Marchal, “THING: introducing a tablet-based interaction technique for controlling 3d hand models," in Proceedings of 33rd Annual ACM Conference on Human Factors in Computing Systems, New York, NY, USA, May 2015.

[24] J. Cui, Y. Zhang, Z. Cai, A. Liu, and Y. Li, "Securing display path for security-sensitive applications on mobile devices," Computers Materials and Continua, vol. 55, no. 1, pp. 17-35, 2018.

[25] C. Yin, J. Xi, R. Sun, and J. Wang, "Location privacy protection based on differential privacy strategy for big data in industrial internet-of things," IEEE Transactions on Industrial Informatics, vol. 14, no. 8, pp. 3628-3636, 2017.

[26] J. Wang, C. Ju, H.-J. Kim, R. Simon Sherratt, and S. Lee, “A mobile assist coverage hole patching scheme based on particle swarm optimization for WSNs," Cluster Computing, vol. 1, pp. 1-9, 2017.

[27] Y. Tu, Y. Lin, J. Wang et al., "Semi-supervised learning with generative adversarial networks on digital signal modulation classification," Computers Materials \& Continua, vol. 55, no. 2, pp. 243-254, 2018. 


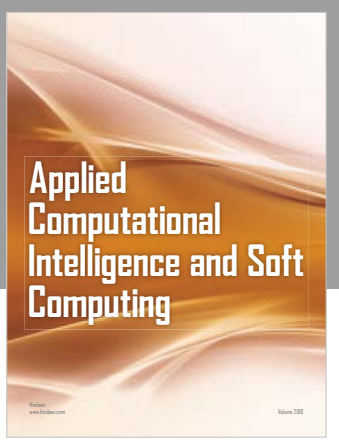

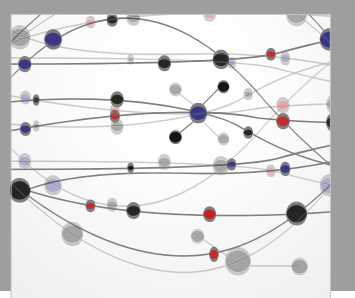

The Scientific World Journal
Submit your manuscripts at

Computing
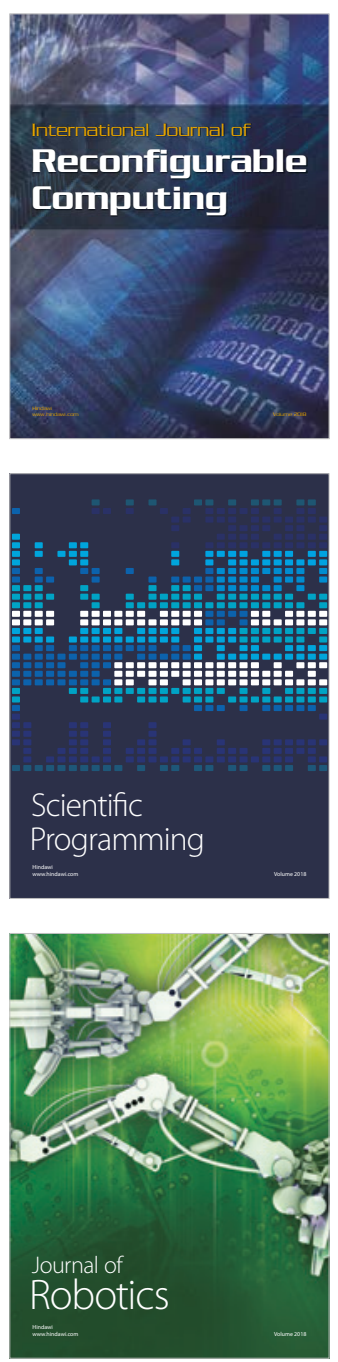

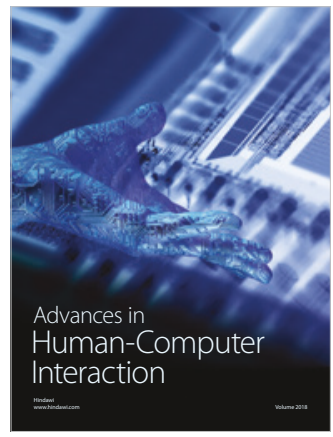

Human-Compute

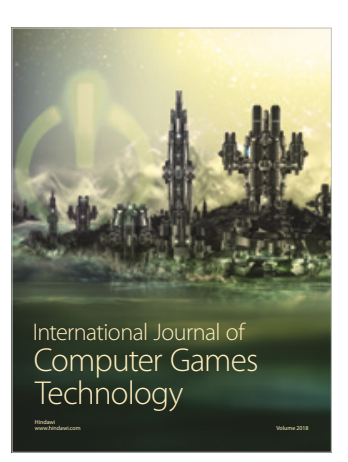

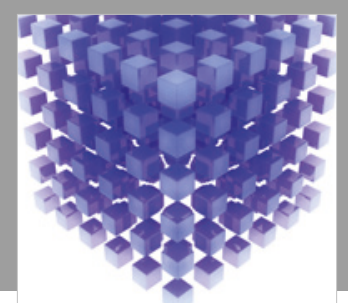

Mathematical Problems in Engineering

\section{Engincering}
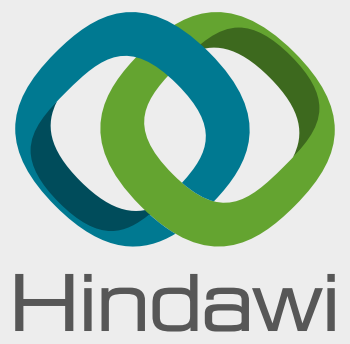

www.hindawi.com
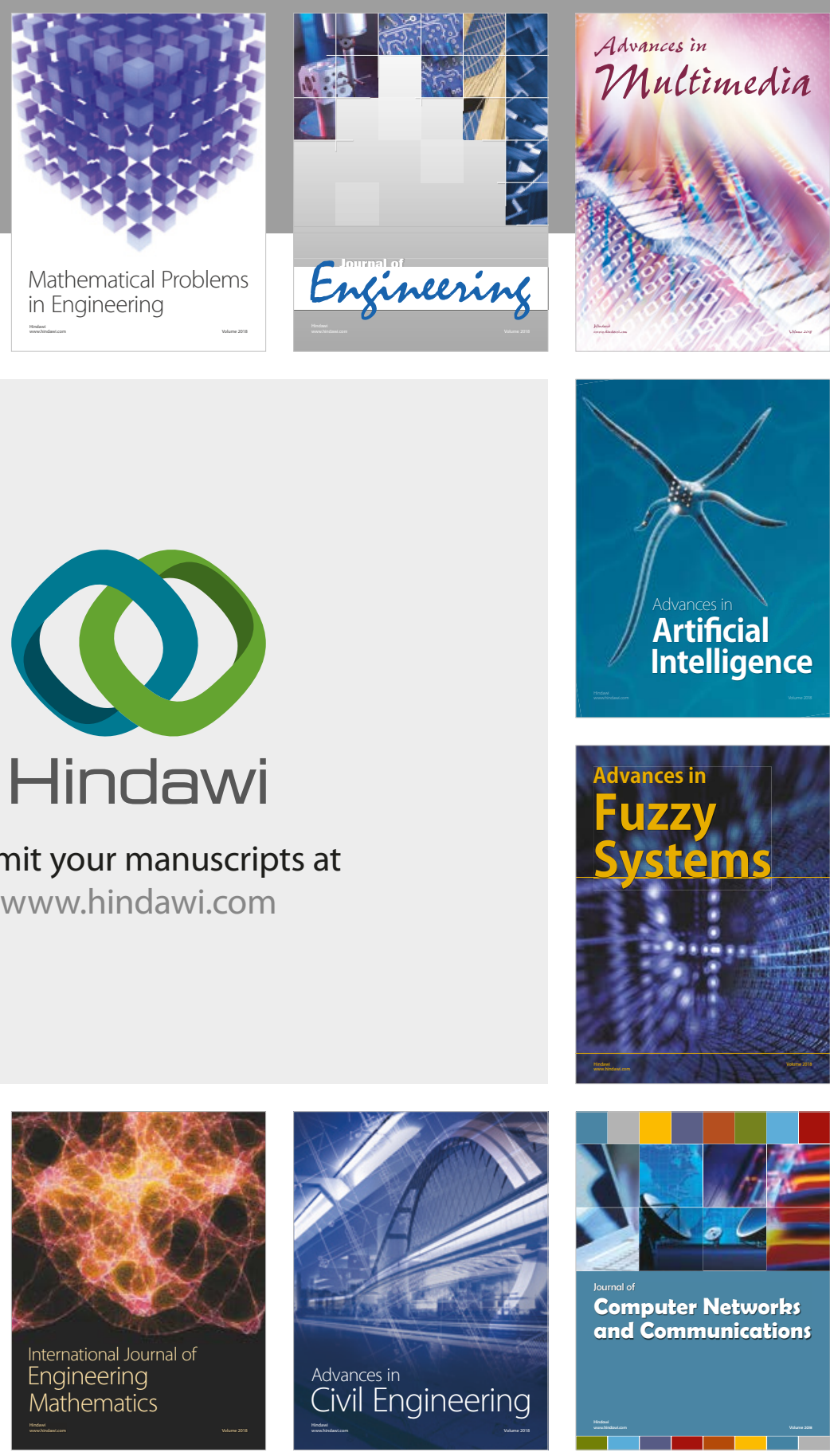

Computer Networks and Communications

Multimedia
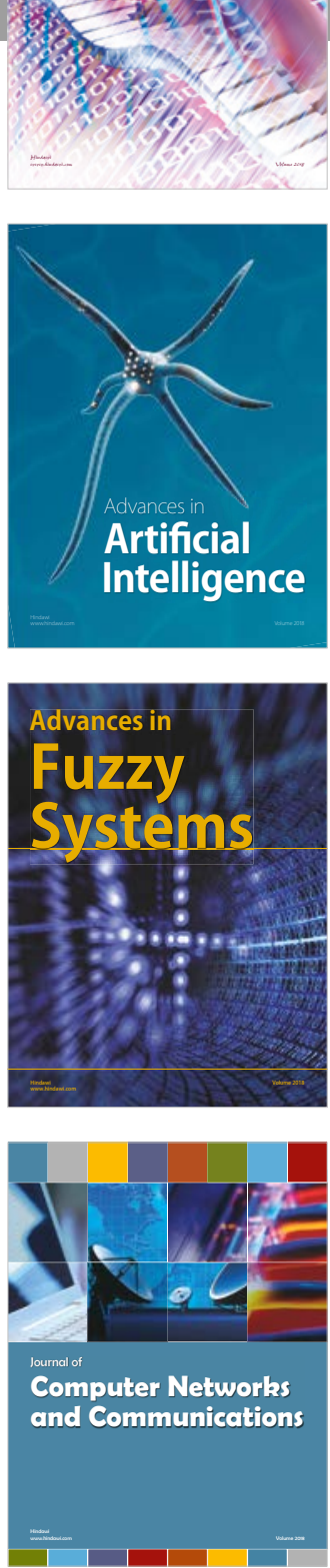

Advances in

Modelling \&

Simulation

in Engineering

interaction

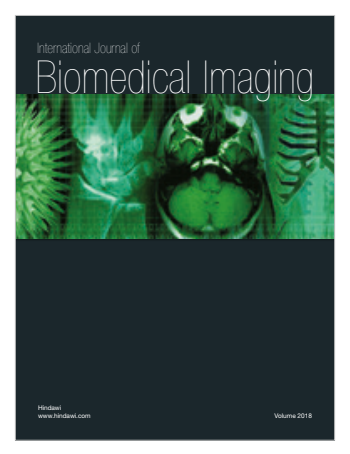

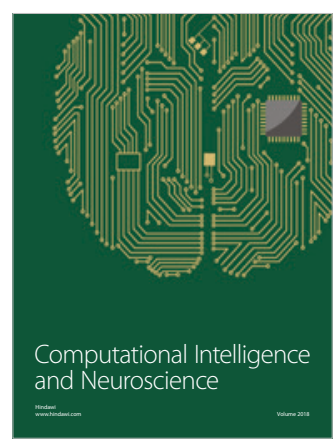

